\title{
Binder migration during drying of lithium-ion battery electrodes: modelling and comparison to experiment
}

\author{
F. Font ${ }^{\mathrm{a}, \mathrm{b}}$, B. $\operatorname{Protas}^{\mathrm{b}}$, G. Richardson ${ }^{\mathrm{c}}$, J. M. Foster ${ }^{\mathrm{d}}$ \\ ${ }^{a}$ Department of Physics, Universitat Politècnica de Catalunya, Barcelona, Spain \\ ${ }^{b}$ Department of Mathematics and Statistics, McMaster University, Hamilton, ON, \\ Canada \\ ${ }^{c}$ Mathematical Sciences, University of Southampton, Hampshire, UK \\ ${ }^{d}$ School of Mathematics, University of Portsmouth, Hampshire, UK
}

\begin{abstract}
The drying process is a crucial step in electrode manufacture that may lead to spatial inhomogeneities in the distribution of the electrode components resulting in impaired cell performance. Binder migration during the drying process, and the ensuing poor binder coverage in certain regions of the electrode, can lead to capacity fade and mechanical failure (e.g. electrode delamination from the current collector). A mathematical model of electrode drying is presented which tracks the evolution of the binder distribution, and is applicable in the relatively high drying rates encountered in industrial electrode manufacture. The model predicts that constant low drying rates lead to a favourable homogeneous binder profiles, whereas constant high drying rates are unfavourable and result in accumulation of binder near the evaporation surface and depletion near the current collector. These results show strong qualitative agreement with experimental observations and provide a cogent explanation for why fast drying conditions result in poorly performing electrodes. Finally, a scheme is detailed for optimisation of a time-varying drying procedure that allows for short drying times whilst simultaneously ensuring a close to homogeneous binder distribution throughout the electrode.
\end{abstract}

Keywords: Electrode drying; binder migration; mass transfer: lithium-ion battery; 


\section{Introduction}

Lithium-ion batteries are currently used to power the vast majority of portable electronic devices, such as cell-phones, laptops, and tablets, and are growing in popularity for use in hybrid and electric vehicles [1]. While one of the biggest challenges in lithium-ion battery research is to increase the energy density of batteries, another equally important challenge is to optimize the manufacturing process to improve long-term cycling performance and capacity lifetime while keeping control of the manufacturing costs $[2,3,4]$. One particularly sensitive step in cell production, which determines the final quality of the battery pack, is the manufacturing process for the electrodes $[5,6]$.

Typically, electrodes are manufactured by coating a current collector with a slurry mixture comprised of active material (AM) particles, conductive carbon nanoparticles, polymer binder (commonly polyvinylidene fluoride (PVDF)) and solvent (commonly N-Methyl-2-pyrrolidone (NMP)) [7, 8, 5, 6]. This mixture is then dried (i.e. the solvent evaporated) by exposure to one or more of the following: air flow, heat, a reduction in ambient pressure or radiation $[5,6,9,10,11]$. State-of-the-art industrial processes typically rely on convective impingement driers which dry films in only a few minutes [12]. The mixture preparation and coating steps prior to drying have to be carefully executed in order to ensure good electrode performance. For instance, it has been shown that slurry mixtures prepared by a multi-step process lead to a more uniform distribution of AM and carbon particles, resulting in less electrode polarization and better cycling capability $[7,8]$. In addition, since the slurry is often stored for considerable periods of time before drying, it must be stable to sedimentation, particularly of the relatively large electrode particles. Such stabilization may arise from various causes including repulsive electrostatic interactions or the formation of a polymer gel state [13]. For an extensive review on mixture preparation the reader is referred to [14].

The most frequently used coating method in industry is slot-die coating, in which a liquid is poured into a die that deposits the coating liquid onto a rolling substrate belt. Coating defects such as film instability and edge effects can occur and need to be controlled which can, e.g. , be achieved by varying the coating speed and the gap ratio $[15,16]$. In contrast, many research-grade electrodes are manufactured by manually spreading the slurry on the substrate using a doctor blade. The use of NMP as a solvent is also highly costly and replacing it with aqueous solutions would both reduce the 
cost of electrode production and be more environmental friendly [17].

Drying begins once the current collector has been coated with the wet particulate electrode mixture. The AM particles are in suspension in the mixture whilst the binder is dissolved in the solvent. In the first stage of the drying process (film shrinkage) solvent evaporates from the top surface of the film causing it to shrink, until the rigid AM particles make contact with each other stopping any further decrease in film thickness. This initiates the second stage of drying (pore emptying) in which the solvent in the pores between the AM particles evaporates. Transport of binder in the film shrinkage stage is believed to be more significant than in the pore emptying stage $[6,12]$ because, during pore emptying, capillary effects lead to a disconnection of the pore network (large pores empty before smaller ones) and consequently to the development of isolated regions of solvent.

Electrode drying has been the subject of intense experimental research in recent years $[5,6,9,18,19,12,20,21,22]$ and a consensus has developed that changes to the drying process parameters (temperature, air-flow, pressure and radiation intensity) significantly affect the final electrode microstructure and hence the electrochemical and mechanical properties of the electrode. High temperatures and drying rates have been observed to lead to accumulation of binder at the evaporation surface and corresponding depletion close to the current collector $[5,9,19,20]$. The consequences of a non-uniform binder distribution include lower adhesion of the electrode to the current collector $[5,6,9,20]$, increased electrical resistivity [5, 22] and decreased cell capacity [6]. Chou et al [23] conclude that even though binder makes up only a small fraction of the electrode composition, it plays a very important role in the cycling stability and rate capability of the electrode. In a recent experimental investigation of the effects of drying on binder distribution by Jaiser et al [6] it was suggested that the electrode film consolidates by the formation of a dense layer of electrode particles, or 'crust', on the drying surface which then grows down until it reaches the current collector. This hypothesis has since been contradicted by the results of Forouzan et al [21] and by a follow-up study by Jaiser et al [12] which both indicate that, as film shrinkage occurs, the electrode particles remain homogeneously distributed.

In both [6] and [12] it was found that rapid removal of the solvent causes an enrichment of binder in the upper regions that cannot be compensated by diffusion at high drying rates. We note also the work of Stein et al. [22], on very slow drying of cathode films, which shows drying rate dependence of the binder distribution. 


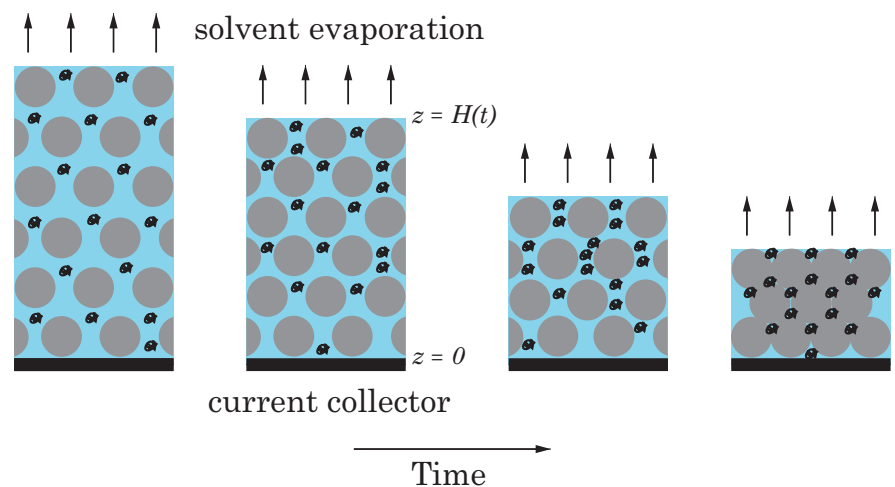

Figure 1: Illustration of the drying process of an electrode film sitting on top of a current collector. The blue background represents the solvent (which evaporates from the top surface of the film), and the black and grey particles represent the polymer binder and AM particles respectively.

Theoretical models detailing the physical mechanisms governing the drying of single-component and two-component colloidal suspensions are studied in $[24,25]$ and $[26,27]$, respectively, while drying of polymer solutions is investigated in $[28,29]$. However, to the best of our knowledge, the process of (industrially relevant) fast drying suspensions composed of electrode particles in a binder solution has not been properly tackled before ${ }^{1}$. The aims of this work are to: (i) model this process, (ii) to compare the model predictions to experimental results, and (iii) to use the model to predict optimal drying strategies. In the next section we formulate and solve a simple model for the mass transport of solvent and binder during the film shrinkage stage of electrode drying. In agreement with the results in [12] we take the electrode slurry to be stable to sedimentation of the electrode particles. In $\S 3$, we present solutions to the model, in realistic parameter regimes and for different drying rates and protocols, before discussing techniques for optimising the drying process. Finally, in $\S 4$ we draw our conclusions.

\footnotetext{
${ }^{1}$ Stein et al. [22] present some modeling results but their relevance to electrode slurries is not entirely clear because volume fractions of the different phases are not specified properly.
} 


\section{Problem formulation}

We formulate a one-dimensional model in which mass transfer occurs only in the $z$-direction (perpendicular to the substrate). All model equations are defined for $z \in[0, H(t)]$, where $H(t)$ is the position of the top of the electrode film, see Figure 1. We track two material phases: a liquid phase (both solvent and dissolved binder), with volume fraction $\phi_{l}$, and a solid phase (AM particles), with volume fraction $\phi_{\mathrm{s}}$. The highly electronically conductive carbon black additive (which makes up only a very small fraction of the electrode volume at around 1\%) is not treated as a separate phase. Instead, its presence is accounted for by incorporating its volume into that of the binder.

We assume no spatial gradients in temperature throughout the film, justifying this assumption by noting that the least (thermally) conductive constituent of the film, the NMP solvent, has a thermal diffusivity of around $\alpha=\lambda / c_{p} \rho \sim 10^{-7} \mathrm{~m}^{2} \mathrm{~s}^{-1}$ (where $\lambda, c_{p}$ and $\rho$, represent the thermal conductivity, heat capacity and density of NMP respectively [30, 31, 32]). This leads to an estimate of the time required to reach thermal equilibrium of around $0.1 \mathrm{~s}$ which is very much shorter than the $(\sim 1 \mathrm{~min})$ drying timescale (the addition of the more conductive materials only serves to further justify this assumption).

As discussed in [13], electrode slurries are generally stabilised so as to stop the sedimentation and aggregation of AM particles. This leads us to expect the AM particles to be uniformly distributed throughout the electrode film during the drying process, an assumption that is borne out by the images of particle distributions at different stages of drying presented in [12]. We do not specify the exact physical mechanism responsible for stabilisation, noting that it could be electrostatic repulsion or as a result of a polymer gel state.

It remains to specify a model for the transport of the PVDF binder particles. These particles are typically very small and therefore are largely unaffected by gravitational buoyancy effects over the drying time of the film. Nevertheless, it seems conceivable that they may be entirely stabilised, like the electrode particles, and, in order to counter this hypothesis, we note that experiments conducted in $[6,12]$ show the binder distribution is strongly dependent on drying rate. Moreover, we note that PVDF is partly chosen as a binder because it is relatively chemically inert and is believed to only form bonds via relatively weak van der Waals interactions [33, 23]. We therefore

model transport of PVDF within the NMP solvent by an advection-diffusion 
equation capturing both the effect of the viscous drag owing to the drying flows (i.e., motion of the solvent driven by evaporation) and mass diffusion. Changes in the macroscopic (effective) diffusivity of the binder (caused by changes in the volume fractions of the phases) will be accounted for via use of the Bruggemann approximation [34]. Finally, we note that PVDF crystallisation only begins to occur when the mass fraction of the binder reaches $77 \mathrm{wt} \%$ at $60^{\circ} \mathrm{C}$ (or larger for higher temperatures) [35]. The parameters in Table 1 indicate that these concentrations will not occur during the film shrinkage stage of the drying process unless an extremely aggressive drying rate is used (see §3.3). They will occur later during the pore emptying stage, but since the binder distribution is already largely determined at that time, crystallisation effects need not be considered in the model.

The model is formulated by coupling conservation equations for the mass transport of the liquid (dissolved binder and solvent) and solid (AM particles) phases to an advection-diffusion equation for the transport of binder through the moving solvent. We can adopt this approach because the volume fraction of binder is so small (typically only $0.01-0.05[8,5,6,12]$ ) that it does not significantly influence the mass transport of the other phases. This in turn implies that the advection-diffusion equation for binder transport decouples from the other transport equations allowing the mass transport equations (for the liquid and solid phases) to be solved (without reference to the binder distribution) prior to solving the equation for the binder concentration accounting for the advective effects of the previously calculated solvent flow.

\subsection{Mass transport}

The mass conservation equations for the solid (AM particles) and liquid (binder and solvent) phases expressed in terms of their volume fractions $\phi_{\mathrm{s}}$ and $\phi_{l}$ are

$$
\begin{aligned}
& \frac{\partial \phi_{\mathrm{s}}}{\partial t}+\frac{\partial F_{\mathrm{s}}}{\partial z}=0 \\
& \frac{\partial \phi_{l}}{\partial t}+\frac{\partial F_{l}}{\partial z}=0
\end{aligned}
$$

where $F_{l}$ and $F_{\mathrm{s}}$ are, respectively, the liquid and solid volume fraction fluxes (dimensions of $\mathrm{m} / \mathrm{s}$ ), which relate to the corresponding volume-averaged ve- 
locities $v_{\mathrm{s}}$ and $v_{l}$ via

$$
\begin{gathered}
F_{\mathrm{s}}=v_{\mathrm{s}} \phi_{\mathrm{s}}, \\
F_{l}=v_{l} \phi_{l} .
\end{gathered}
$$

In addition, the volume fractions of the two phases (liquid and solid) satisfy

$$
\phi_{\mathrm{s}}+\phi_{l}=1 \text {. }
$$

In order to model the stabilisation of the electrode particles in the slurry we assume that they are uniformly distributed

$$
\phi_{\mathrm{s}}=\phi_{\mathrm{s}}(t)
$$

a hypothesis that is confirmed by the experimental particle distribution images contained in [12].

The equations above are solved subject to no-flux boundary conditions on the current collector, namely,

$$
\begin{aligned}
& \left.F_{\mathrm{s}}\right|_{z=0}=0, \\
& \left.F_{l}\right|_{z=0}=0,
\end{aligned}
$$

and the following flux conditions on the evaporation surface $z=H(t)$ :

$$
\begin{aligned}
& \left.\frac{d_{\mathrm{s}}}{d t}(z-H)\right|_{z=H(t)}=0 \quad \Longrightarrow \quad-\dot{H}+\left.v_{\mathrm{s}}\right|_{z=H(t)}=0, \\
& \left.\frac{d_{l}}{d t}(z-H)\right|_{z=H(t)}=\left.\frac{\gamma}{\phi_{l}}\right|_{z=H(t)} \quad \Longrightarrow \quad-\dot{H}+\left.v_{l}\right|_{z=H(t)}=\left.\frac{\gamma}{\phi_{l}}\right|_{z=H(t)},
\end{aligned}
$$

which represent zero-flux of the solid phase and an evaporation flux $\gamma$ of the liquid phase, respectively, through the surface $z=H(t)$. In (6) and (7) the operators $d_{\mathrm{s}} / d t$ and $d_{l} / d t$ are material derivatives taken with respect to the solid and liquid velocities, respectively, and a dot indicates a derivative with respect to time. At the beginning of the drying process we assume that the two phases are well mixed and are present in the following proportions

$$
\begin{aligned}
& \left.\phi_{\mathrm{s}}\right|_{t=0}=\phi_{\mathrm{s}}^{0}, \\
& \left.\phi_{l}\right|_{t=0}=1-\phi_{\mathrm{s}}^{0},
\end{aligned}
$$

whilst the film is taken to have initial thickness

$$
\left.H\right|_{t=0}=H_{0}
$$


Validity of the model terminates at the time $t_{\text {end }}$ when the AM particles are consolidated (i.e. they make direct contact with each other) and the pore emptying phase of the drying begins. We define the solid volume fraction at this fully consolidated stage to be $\phi_{\mathrm{s}}^{\max }=\phi_{\mathrm{s}}\left(t_{\mathrm{end}}\right)$ and model solutions will be terminated when this state is reached.

Summing equations (1a)-(1b), using (3), integrating with respect to $z$ and imposing the boundary conditions (5) reveals that

$$
F_{l}+F_{\mathrm{s}}=0 .
$$

Substituting the above into the sum of the boundary conditions (6) and (7), and using (2) and (3), gives the following evolution equation for the position of the top surface of the film

$$
\dot{H}=-\gamma
$$

This result can readily be interpreted as global mass conservation throughout the film.

As illustrated in Figure 1, and evidenced in [12], the solid volume fraction is space-independent because of the stable nature of the suspension. Thus, equation (1a) can be integrated with respect to $z$ and (5a) imposed to give

$$
F_{\mathrm{s}}=-\dot{\phi}_{\mathrm{s}} z
$$

Eliminating $F_{\mathrm{s}}$ from the above in favour of $v_{\mathrm{s}}$ using $(2 \mathrm{a})$ and using the boundary condition (6) gives $d / d t\left(H \phi_{\mathrm{s}}\right)=0$. This can be integrated and the initial conditions (8a) and (9) imposed to give $\phi_{\mathrm{s}}=H_{0} \phi_{\mathrm{s}}^{0} / H$. Back substitution of this result into (12), then using (10) and (11) gives the following expressions for the volume fractions and volume-averaged fluxes

$$
\phi_{\mathrm{s}}=\frac{\phi_{\mathrm{s}}^{0} H_{0}}{H}, \quad \phi_{l}=1-\frac{\phi_{\mathrm{s}}^{0} H_{0}}{H}, \quad F_{\mathrm{s}}=-\frac{\gamma \phi_{\mathrm{s}}^{0} H_{0}}{H^{2}} z, \quad F_{l}=\frac{\gamma \phi_{\mathrm{s}}^{0} H_{0}}{H^{2}} z .
$$

\subsection{Binder transport}

The polymer binder is distributed within the liquid phase only, thus, a volume-averaged continuity equation describing the concentration $c$ of dissolved binder in the solvent is

$$
\frac{\partial}{\partial t}\left(\phi_{l} c\right)+\frac{\partial J}{\partial z}=0, \quad J=F_{l} c-D_{\text {eff }} \frac{\partial c}{\partial z},
$$


where $J$ is the volume-averaged mass flux of dissolved binder. This flux is composed of two parts: an advective part with the volume-averaged velocity of the solvent and a diffusive part with an "effective" diffusion coefficient $D_{\text {eff }}$. We estimate this effective diffusivity using the Bruggemann approximation which assumes that $D_{\text {eff }}=D \phi_{l}^{3 / 2}$, where $D$ is the diffusivity of the binder in the solvent [36]. Notably, the value of $D_{\text {eff }}$ decreases during the drying process, as $\phi_{l}$ varies according to (13), representing the increasing tortuosity of the solid network.

Suitable boundary conditions on (14) require that there is zero flux of binder through both the current collector and the free surface $z=H(t)$. We therefore have

$$
\left.J\right|_{z=0}=0,\left.\quad J\right|_{z=H(t)}=\phi_{l} c \frac{d H}{d t} .
$$

One can verify that the total amount of binder in the film $\int_{0}^{H(t)} c \phi_{l} d z$ is conserved throughout the drying process. We assume that initially the binder is homogeneously distributed throughout the solvent. Thus, a suitable initial condition to close (14) is

$$
\left.c\right|_{t=0}=c_{0} .
$$

\subsection{Parameter estimates}

We calibrate simulations based on the experiments conducted in [6, 12], and summarised here in Table 1, for industrially relevant electrode slurries and drying protocols. We find that the initial volume fraction of the solid electrode particles is

$$
\phi_{\mathrm{s}}^{0}=\frac{\omega_{\mathrm{s}}^{0}}{\rho_{\mathrm{s}}}\left(\frac{\omega_{\mathrm{s}}^{0}}{\rho_{\mathrm{s}}}+\frac{\omega_{\mathrm{b}}^{0}}{\rho_{\mathrm{b}}}+\frac{\omega_{\mathrm{NMP}}^{0}}{\rho_{\mathrm{NMP}}}\right)^{-1} \approx 0.2792 .
$$

where the subscripts "s", "b" and "NMP" indicate solid electrode particles, binder and solvent, respectively. To compute the final (and maximal) value of the solid volume fraction we also make use of the measured porosity of the dried electrode film $p=0.46[12]$ and find that

$$
\phi_{\mathrm{s}}^{\max }=(1-p) \frac{\omega_{\mathrm{s}}^{\max }}{\rho_{\mathrm{s}}}\left(\frac{\omega_{\mathrm{s}}^{\max }}{\rho_{\mathrm{s}}}+\frac{\omega_{\mathrm{b}}^{\max }}{\rho_{\mathrm{b}}}+\frac{\omega_{\mathrm{NMP}}^{0}}{\rho_{\mathrm{NMP}}}\right)^{-1} \approx 0.5032 .
$$


The initial concentration of binder in the solvent and film thickness are

$$
c_{0}=\frac{\tilde{c}_{0}}{\left(1-\phi_{\mathrm{s}}^{0}\right)} \approx 0.057 \mathrm{~mol} / \mathrm{m}^{3}, \quad H_{0} \approx 114 \mu \mathrm{m},
$$

where $\tilde{c}_{0}=\rho w_{\mathrm{b}}^{0} / M_{b}=0.041 \mathrm{~mol} / \mathrm{m}^{3}$ with $\rho=\rho_{\mathrm{s}} w_{\mathrm{s}}^{0}+\rho_{\mathrm{b}} w_{\mathrm{b}}^{0}+\rho_{\mathrm{NMP}} w_{\mathrm{NMP}}^{0}$ and $M_{b}=1000 \mathrm{~kg} / \mathrm{mol}$ (PVDF molecular weight; Solvay Solef 5130) is the initial concentration of binder in the film $[6,18,12]$.

We base our estimates of the drying rate on the experiments presented in [6] where an impingement dryer is used to dry films to the point where their thickness is reduced to its final value in around 1 minute, in line with typical industrial drying rates. This leads to a representative value of the mass flux across the evaporation surface of $q_{\mathrm{s}}=1.19 \mathrm{~g} \mathrm{~m}^{-2} \mathrm{~s}^{-1}$, and a corresponding value for the evaporation rate of

$$
\gamma=\frac{q_{\mathrm{s}}}{\rho_{\mathrm{NMP}}} \approx 1.16 \times 10^{-6} \mathrm{~m} \mathrm{~s}^{-1} .
$$

Other common techniques found in the literature include air drying [26], exposure to infrared radiation [11] and the use of a vacuum oven [21,22]. While some of these can result in drastically different drying times the vacuum oven technique used in Stein et al. [22] led to a film shrinkage time of around 1 minute (3 minutes) when a temperature of $120^{\circ} \mathrm{C}\left(70^{\circ} \mathrm{C}\right)$ was used which is in line with our estimates.

The PVDF diffusion coefficient $D=1.14 \times 10^{-10} \mathrm{~m}^{2} / \mathrm{s}$ is obtained, as described in the supplementary information, by qualitatively matching the model solutions to the experimental results presented in [6]. We note that it is reasonable to expect this quantity to be dependent on the concentration of PVDF. However, due to the lack of experimental data with which to tune this functional dependence, we opt to make the simplest assumption and take it to be constant. Some of the possible effects associated with the relaxation of this assumption are discussed in $§ 3.5$.

\section{Results and discussion}

In this section we present and contrast typical model solutions for both relatively high and low drying rates. Then, we reproduce the experimental procedure followed in [6] and demonstrate good agreement between model solutions and experimental results. Finally, we examine the effects of allowing time-dependent drying rates and consider how this can be used to devise possible strategies to optimize the drying process. 


\begin{tabular}{|c|c|c|c|c|}
\hline \hline Material & $\rho\left(\mathrm{g} / \mathrm{cm}^{3}\right)$ & $\omega^{0}$ & $\omega^{\max }$ & $\mu(\mathrm{mPas})$ \\
\hline Solvent (NMP) & 1.03 & 0.525 & 0 & 0.85 \\
Polymer binder (PVDF) & 1.76 & 0.026 & 0.055 & - \\
Graphite particles & 2.21 & 0.449 & 0.945 & - \\
\hline \hline
\end{tabular}

Table 1: Typical densities $\rho$ and viscosity $\mu$ for film components and initial and final mass fractions, respectively, $\omega^{0}$ and $\omega^{\max }$, according to Jaiser et al $[6,12]$. Our modelling approach follows that used in Forouzan et al [21] in the sense that we do not track a separate carbon black phase. Instead, its mass fraction $(0.014[6,12])$ has been incorporated to the mass fraction of the PVDF binder.

\subsection{The differences between low and high drying rates}

The evolution of the binder distribution is determined by solving (14)(16) where the phase volume fractions and fluxes are given by (13). Although no exact solutions to this problem are available, we can solve the problem approximately in two different ways: (i) using matched asymptotic expansions valid for limiting values of the Peclet number $P e=\gamma H_{0} / D$ (i.e., $P e \gg 1$ and $P e \ll 1$ ), and; (ii) using a numerical scheme based on finite difference approximations. Details on the derivation of the asymptotic solutions and the numerical scheme can be found in the supplementary information. The two methods provide a mutual check on accuracy and while the numerical method is accurate for all values of $P e$ the asymptotic solutions illustrate the dominant physical effect(s) occurring in the different regimes. Further the numerical scheme offers the possibility of solving generalised models, e.g., when the PVDF diffusivity depends on its concentration. We now briefly describe the asymptotic solutions in low drying rate regime (LDR), with $P e \ll 1$, and high drying rate regime (HDR), with $P e \gg 1$.

In the LDR limit $(P e \ll 1)$ the binder concentration is approximated by

$$
\frac{c(z, t)}{c_{0}} \approx \frac{\left(1-\phi_{\mathrm{s}}^{0}\right)}{\left(\frac{H}{H_{0}}-\phi_{\mathrm{s}}^{0}\right)}+P e \frac{\left(1-\phi_{\mathrm{s}}^{0}\right)}{\left(\frac{H}{H_{0}}-\phi_{\mathrm{s}}^{0}\right)^{2} \phi_{l}(t)^{1 / 2}}\left(\frac{z^{2}}{2 H_{0}^{2}}-\frac{H^{2}}{6 H_{0}^{2}}\right) .
$$

where $H(t)=H_{0}-\gamma t$ (obtained after integrating (11)). The solution (21) is uniform up to leading order in the Peclet number showing that diffusion effects are sufficiently strong to maintain an almost uniform binder distribution throughout the film.

In the HDR limit $(P e \gg 1)$ the binder concentration can be approximated 
by

$$
\frac{c(z, t)}{c_{0}} \approx 1+A(t) \exp \left(-\frac{P e(H-z)}{H_{0} \phi_{l}(t)^{3 / 2}}\right),
$$

where the function of integration $A(t)$ is a solution to the following initialvalue problem

$$
\dot{A}=-\frac{5}{2} A \frac{\dot{\phi}_{l}(t)}{\phi_{l}(t)}+\frac{P e}{\phi_{l}(t)^{3 / 2}}, \quad A(0)=1 .
$$

In this regime advection dominates except in a narrow boundary layer, of width of order $H_{0} / P e$, near the evaporation surface where diffusion is also important. It follows, since the upward advection velocity is greater than or equal to zero (it is zero on the current collector), that the binder concentration throughout the bulk of the film just takes its initial uniform value $c_{0}$. The exception to this is in the boundary layer adjacent to the evaporation surface where the binder that has been brought to the surface by advection.

In Figure 2 we compare typical asymptotic solutions for both low and high drying rates to full numerical solution. In particular we consider $\gamma=$ $1.25 \cdot 10^{-7} \mathrm{~m} \mathrm{~s}^{-1}(P e \approx 0.1)$ and $\gamma=1.25 \cdot 10^{-5} \mathrm{~ms}^{-1}(P e \approx 10)$. These are around one order of magnitude larger and smaller than the reference drying rate taken from [6]. The red solid lines correspond to the asymptotic solutions (21)-(23) and blue dashed lines to the numerical solution. The two solution approaches exhibit very favourable agreement. For the LDR the concentration of binder progressively increases as solvent evaporates with the distribution remaining almost homogeneous throughout the whole drying process resulting in a good quality electrode. However the drying time is relatively large. Contrastingly, for the HDR, the drying time is relatively small leading to relatively large solvent velocities with correspondingly large concentration gradients which cannot be dissipated by diffusion. As a result, binder accumulates near the top surface of the electrode film and there is relatively poor coverage in the bulk because of a failure to concentrate binder in this region as the solvent evaporates (as in the LDR).

\subsection{Agreement with experiment}

We now utilise the model to reproduce and elucidate the experimental results obtained in Jaiser et al [6]. In their work electrode films were first dried at a high rate for a given period of time, $t_{\text {trans }}$, after which the rate 
(a) LDR

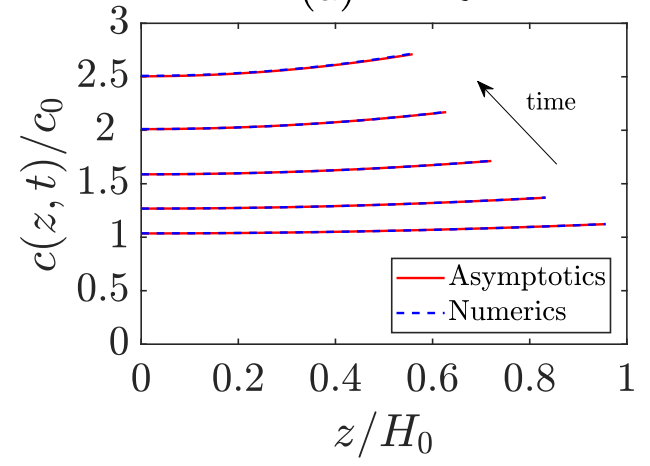

(b) HDR



Figure 2: Numerical and asymptotic solutions of the binder transport model (14)(16). Panel (a) shows concentration profiles corresponding to a low drying rate $(\gamma=$ $1.25 \cdot 10^{-7} \mathrm{~m} \mathrm{~s}^{-1}$ ) for five values of time between $39.8 \mathrm{~s}$ and $401.92 \mathrm{~s}$. Panel (b) shows concentration profiles corresponding to a high drying rate $\left(\gamma=1.25 \cdot 10^{-5} \mathrm{~m} \mathrm{~s}^{-1}\right)$ for five values of time between $0.4 \mathrm{~s}$ and $4.02 \mathrm{~s}$. The last profile in (a) and (b) corresponds to the concentration profile at the end of drying $t=t_{\text {end }}$. Note that panel (b) has been truncated at $z=0.3$ for ease of viewing; the solution for $z<0.3$ is essentially flat, with $c(z, t) / c_{0} \approx 1$.

was decreased and evaporation continued until drying was complete. One of the main results was a plot of $c_{\mathrm{top}}=\left.c\right|_{z=H, t=t_{\text {end }}}$ and $c_{\mathrm{bot}}=\left.c\right|_{z=0, t=t_{\text {end }}}$ (i.e. the binder concentrations at the top (evaporation surface) and bottom (current collector) of the electrode at the end of the drying process $t=t_{\text {end }}$ ) against $t_{\text {trans }}$. They observed that: (i) $c_{\text {bot }}$ is almost constant for sufficiently small $t_{\text {trans }}$, (ii) there is then a small range of values of $t_{\text {trans }}$ where $c_{\text {top }}$ increases whereas $c_{\text {bot }}$ decreases beyond which, (iii) $c_{\text {top }}$ and $c_{\text {bot }}$ saturate to constant values. We reproduce this protocol in our model by taking the time-dependent drying rate used in [6], namely

$$
\gamma(t)=\left\{\begin{array}{lll}
1.16 \times 10^{-6} \mathrm{~m} \mathrm{~s}^{-1} & (\mathrm{Pe}=0.94) & \text { for } t<t_{\text {trans }} \\
0.51 \times 10^{-6} \mathrm{~m} \mathrm{~s}^{-1} & (\mathrm{Pe}=0.41) & \text { for } t>t_{\text {trans }}
\end{array}\right.
$$

Figure 3 shows that $c_{\text {bot }}$ is almost constant until $t_{\text {trans }} \gamma / H_{0} \approx 0.36$ and then decreases until $t_{\text {trans }} \gamma / H_{0} \approx 0.44$. For even larger values of $t_{\text {trans }}$, the decrease in drying rate does not occur until after the electrode is completely dry, thus $c_{\text {bot }}$ remains constant. The evolution of $c_{\text {top }}$ behaves in the opposite fashion; it first remains roughly constant until $t_{\text {trans }} \gamma / H_{0} \approx 0.36$, then increases until $t_{\text {trans }} \gamma / H_{0}=t_{\text {end }} \gamma / H_{0} \approx 0.44$ and stays constant for $t_{\text {trans }}>t_{\text {end }}$. These results show strong qualitative agreement with those presented in Figure 6 
(a)

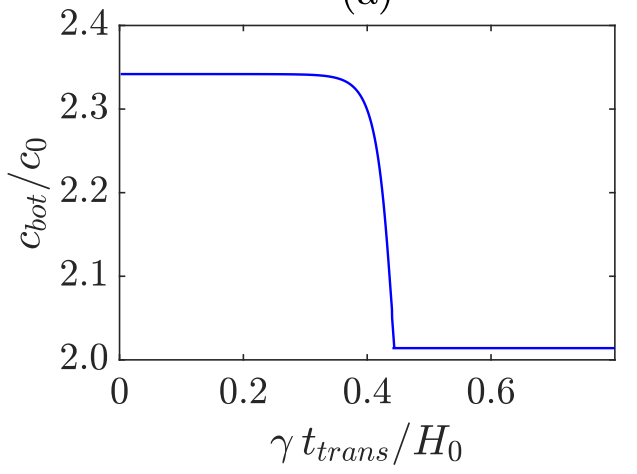

(b)

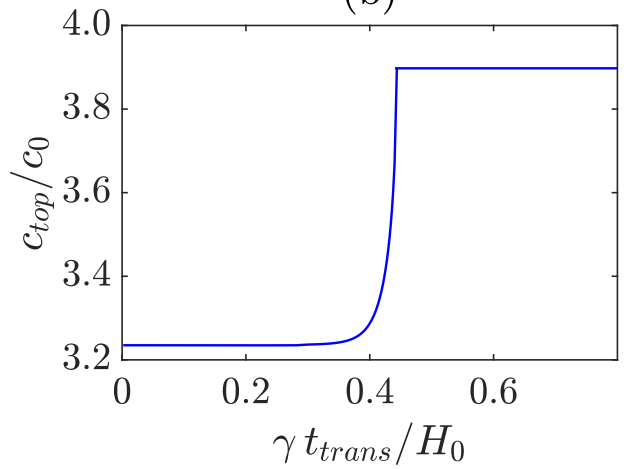

Figure 3: Normalized concentration of binder on top and bottom of the electrode at the end of drying as a function of the nondimensional transition time between high and low drying rate as determined using the numerical scheme described in the supplementary information.

from [6]. We note that there is a small quantitative discrepancy between the experimental and model results. The model predicts that $c_{\text {top }}$ should saturate for very small values of $t_{\text {trans }}$ whereas the experiment shows this value decreasing until $t_{\text {trans }}=0$. One possible explanation for this is that the diffusivity of PVDF decreases where binder concentrations are large. If this were the case then the accumulation of binder near the surface, caused by an initial period of fast drying, would require more time to be dissipated than is suggested by the constant value of $D$ assumed here.

\subsection{Identifying viable constant drying rates}

We now identify the largest rate at which an electrode can afford to be dried without inducing unacceptably large gradients in the binder concentration. Figure 4 shows that the value of the drying rate for which concentration gradients remain relatively small corresponds to $P e=\gamma H_{0} / D \lesssim 1$. For the values of $H_{0}$ and $D$ estimated in $\S 2.3$ this yields $\gamma \lesssim 1 \cdot 10^{-6} \mathrm{~m} / \mathrm{s}$. Figure 4 also shows that, unless a very aggressive drying rate is used, PVDF crystallization will not begin to occur until after the film is fully consolidated.

\subsection{The advantages of using time-dependent drying rates}

Drying should be carried out slowly to prevent an undesirable accumulation of binder near the evaporation surface. However, from an industrial point of view, short drying processes are preferred in order to increase throughput 




Figure 4: Binder concentration on top of the electrode at the end of drying as a function of the drying rate $\gamma$. The solid line corresponds to the numerical solution and the the dashed and dash-dotted lines correspond to the asymptotic solution for low and high drying rate, respectively. The horizontal line denotes the binder concentration at which crystallisation begins to occur at $T=60^{\circ} \mathrm{C}$, corresponding to $c(z, t) / c_{0}=22.75$ [35], which represents an orientative upper bound for the concentration in our model.

[17]. The model is now used to investigate whether (and to what extent) allowing time-dependent drying rates can be helpful in simultaneously achieving more homogeneous binder distributions and shorter drying times. To do so, we consider three different drying protocols: (Case 1) a constant drying rate, (Case 2) a linearly increasing drying rate, and (Case 3) a linearly decreasing drying rate, as outlined below:

$$
\begin{array}{lll}
\text { Case 1: } & \gamma(t)=\gamma_{0} & \text { for } 0 \leq t \leq t_{\text {end }}, \\
\text { Case 2: } & \gamma(t)=2 \gamma_{0} \frac{t}{t_{\text {end }}} & \text { for } 0 \leq t \leq t_{\text {end }}, \\
\text { Case 3: } & \gamma(t)=2 \gamma_{0}\left(1-\frac{t}{t_{\text {end }}}\right) & \text { for } 0 \leq t \leq t_{\text {end }},
\end{array}
$$

and select $\gamma_{0}=1.16 \mu \mathrm{m} \mathrm{s}^{-1}$. Note that in defining (25)-(27) we have ensured that the time taken to fully consolidate the film, $t_{\text {end }}$, is the same in all three cases (see supplementary information). The solutions are shown in Figure 5.

We observe that choosing a linearly decreasing drying rate (case 3) gives the most evenly distributed concentration whilst choosing a linearly increasing one yields the worst results. This suggests that, if the goal is obtaining an acceptably homogeneous distribution of binder and a short drying time, 
(a)

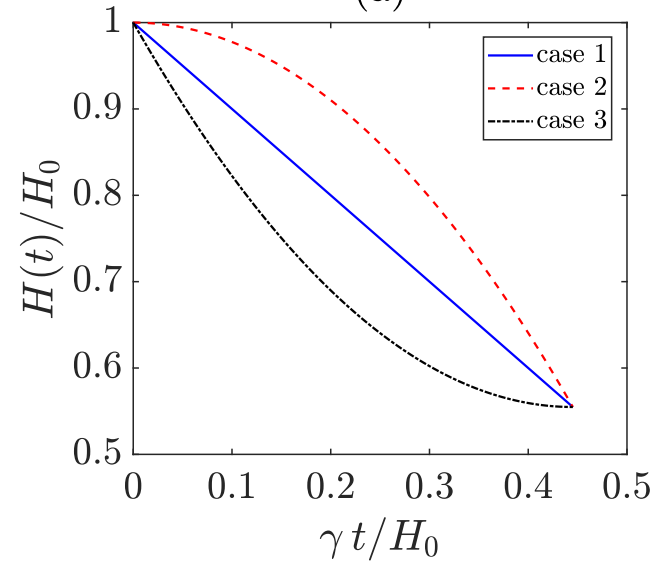

(b)

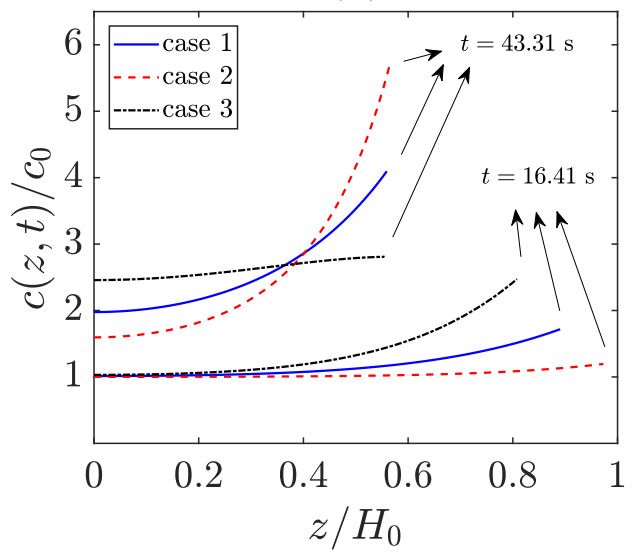

Figure 5: (a) Evolution of the position of the top surface for the three drying rates considered, cf. (25)-(27). (b) Concentration of binder near the beginning $(t=16.41 \mathrm{~s})$ and at the end $(t=43.31 \mathrm{~s})$ of the drying process for each case.

the best procedure is to dry the electrode at a high drying rate at the beginning and at a low drying rate near the end of the process. This can be rationalised by noting that even though large binder concentration gradients may be established by the initial high drying rate, so long as the rate is dropped towards the end of the process, diffusive effects have sufficient time to act to dissipate these inhomogeneities.

We now demonstrate that the strategy of employing a decreasing drying rate can be pushed even further and used to simultaneously decrease total drying times and the magnitude of the binder concentration gradient in comparison to a fixed drying rate. To demonstrate this we solve our model using linearly decreasing drying protocol

$$
\text { Case 4: } \quad \gamma(t)=3 \gamma_{0}\left(1-\frac{t}{t_{\text {end }}^{\prime}}\right) \quad \text { for } \quad 0 \leq t \leq t_{\text {end }}^{\prime} \text {. }
$$

Notably, this leads to the a total drying time which is only two thirds as long as those considered previously in (25)-(27). Figure 6 clearly indicates a more homogeneous binder concentration for protocol (28), thereby confirming that the electrode drying process can be optimized (both in terms of decreasing the total drying time and producing more homogeneous films) by exploiting a carefully chosen time-dependent drying rate. 
(a)

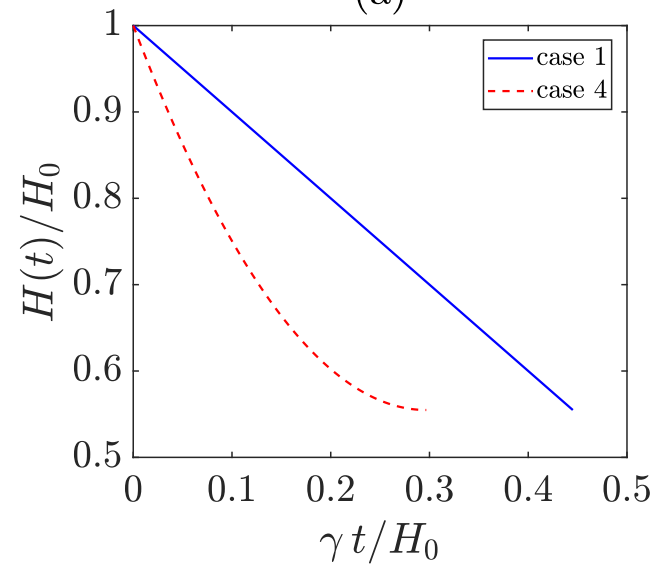

(b)

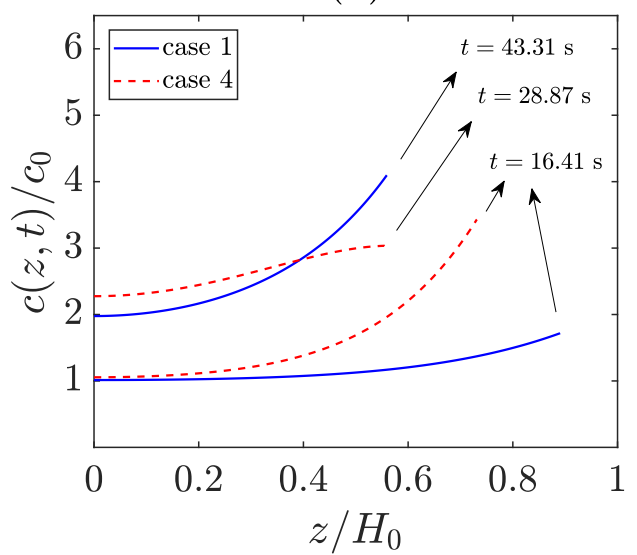

Figure 6: (a) Evolution of the position of the top surface for the drying protocols (25) and (28). (b) Concentration of binder near the beginning $(t=16.41 \mathrm{~s})$ and at the end of the drying process for both protocols. The end time for drying in protocol (28), $t=28.87 \mathrm{~s}$, is shorter than in protocol $(25), t=43.31 \mathrm{~s}$.

\subsection{Optimisation of the drying process}

From a manufacturing stand point perhaps the most important task is to identify a drying protocol that minimises binder inhomogeneities for a given total drying time. We now address this issue by solving an optimal control problem. We restrict our interest to drying rates that are piecewise constant in time, because such rates should be straightforward to realise on practical drying equipment. We split the total drying time into $N$ equally sized subintervals and denote the (constant) evaporation rate during each subinterval $\gamma_{i}$ for $i=1, \ldots, N$, i.e., we take $\gamma(t)=\gamma_{i}$ for $t \in\left(t_{i}, t_{i+1}\right)$ where $t_{i}=(i-1) t_{\text {end }} / N$. The optimal control problem is to identify the vector $\gamma=\left[\gamma_{1}, \ldots, \gamma_{N}\right]$ such that a suitable objective function, which measures the magnitude of the binder concentration gradient, is minimised. More rigorously our aim is to find the optimal set of values for the drying rate on each of the subintervals, denoted by $\gamma^{\text {opt }}$, such that

$$
\gamma^{\text {opt }}=\operatorname{argmin}_{\gamma} \mathcal{J}(\gamma) \quad \text { where } \quad \mathcal{J}=\left.\int_{0}^{H(t)} \frac{\partial c}{\partial x} d x\right|_{t=t_{\text {end }}}=c_{\text {top }}-c_{\text {bot }}
$$

and, $c_{\text {top }}$ and $c_{\text {bot }}$ are, respectively, the binder concentrations at the top and bottom of the film at the end of drying as defined in $§ 3.2$. We note that fixing the total drying time is tantamount to fixing the total amount of solvent that 
is removed from the film over that given window of time. We must therefore solve (29) subject to the constraint that

$$
\int_{0}^{t_{\mathrm{end}}} \gamma(t) d t=\frac{t_{\mathrm{end}}}{N} \sum_{i=1}^{N} \gamma_{i}=H_{0}-H\left(t_{\mathrm{end}}\right) .
$$

Details on the method of solution of this problem are given in the supplementary information.

Concentration-independent diffusivity. First we explore solution of this optimal control problem whilst retaining the assumption that the diffusivity of the binder is independent of its concentration, i.e., that $D_{\text {eff }}=D \phi_{l}^{3 / 2}$ with $D$ a constant. The results in Figure 7 indicate that the optimum strategy is to apply a short period of very fast drying (which causes the binder to accrue at the surface) followed by a quiescent period in which the drying is switched off and the concentration profile is allowed to re-equilibrate under diffusion.

We reiterate that the model only applies to the film shrinkage stage of the drying process and that once this quiescent period has elapsed, drying can be resumed and the pore-emptying stage can begin. Although this drying procedure is optimal, when $D_{\text {eff }}=D \phi_{l}^{3 / 2}$, it is a dangerous one to adopt in practice; if the film is over-dried (beyond the consolidation point where pore emptying begins) before the quiescent period allows the concentration profile to re-equilibrate, the pore emptying and severing of the liquid network prevents the binder redistributing and the unfavourable non-uniform profile will be present in the finished electrode.

The underlying reason for this strategy being optimal is that diffusion is able to more effectively reorganize the concentration once the film height has been reduced and this is facilitated by the rapid initial drying phase. This effect is offset, to a certain extent, by the decrease in $D_{\text {eff }}$ as the film shrinks and the liquid volume fraction $\phi_{l}$ decreases but this is not a strong enough effect to outweigh the enhanced redistribution through the reduced film depth. Importantly, this interpretation suggests that if $D_{\text {eff }}$ decreases with increasing $c$ (even relatively weakly) the optimal drying procedure will be rather different.

Concentration-dependent diffusivity. Here we discuss the possible effects of considering a PVDF solution in which the diffusivity of the polymer decreases with increases in its concentration. Although we were unable to find literature that refers directly to the concentration dependence of PVDF diffusivity 
in NMP, $D=D^{*}(c)$, there is literature (see for example [37]) on generic polymer solutions that suggests that $D^{*}(c)$ is an exponentially decreasing function.

We expect even moderate decreases in $D$ (with increasing $c$ ) to radically change the optimal drying strategy. Our prediction is that an initial rapid drying phase will lead to high polymer binder concentration, and therefore decreased diffusivity, near the evaporation surface which will impede the subsequent redistribution of binder even if the drying rate is decreased in the latter stages of the process. This in turn suggests that where there is a decrease of $D$ with increasing $c$ that a more constant drying protocol, which keeps the binder concentration relatively uniform, will lead to a better polymer distribution than the optimal protocol predicted here for in the constant diffusivity case. This highlights the need for further experimental characterization of the PVDF transport processes in NMP solutions in order to capture more precisely the behavior of $D$ with changes in $c$.

\section{Conclusions}

We have presented a model that predicts the mass transport and evolution of binder concentration during the drying of lithium-ion battery electrodes in cases where the active material particles form a stable suspension on the timescale of the drying process. Although this is not a restriction of the model, we have primarily focused on relatively short drying times that are typical of industrial processes. The motion of the dissolved binder is driven by a combination of mass diffusion and viscous drag owing to the upward flow of the solvent caused by its evaporation on the top surface of the film is moving down, and causing an increase in active material content. We have found that higher drying rates tend to induce larger binder concentration gradients because (i) the more aggressive evaporation rates cause a larger (upward) convection of the binder solvent and (ii) the decreasing drying time allows less opportunity for Brownian motion to redistribute the binder more evenly throughout the film. We have demonstrated that, despite a number of simplifying assumptions, the model satisfactorily reproduces recently published experimental results of binder migration phenomena during drying. Finally, we have shown that a sound strategy to reduce the drying time whilst simultaneously maintaining small variations in the binder concentration is to initially apply a period of high drying rate and to then decrease this rate towards the end of the process. 
(a)

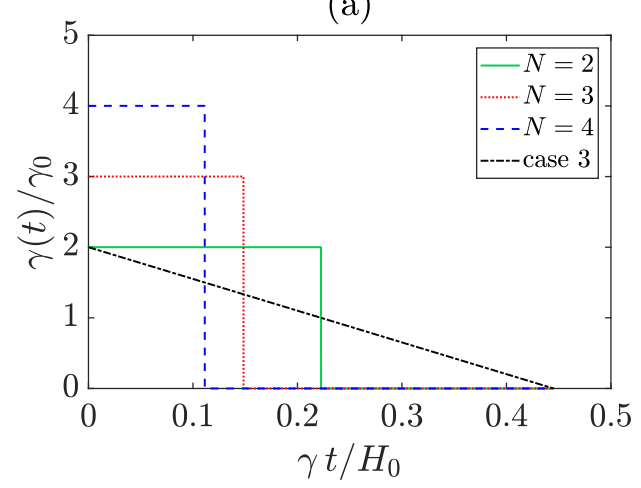

(c)



(b)

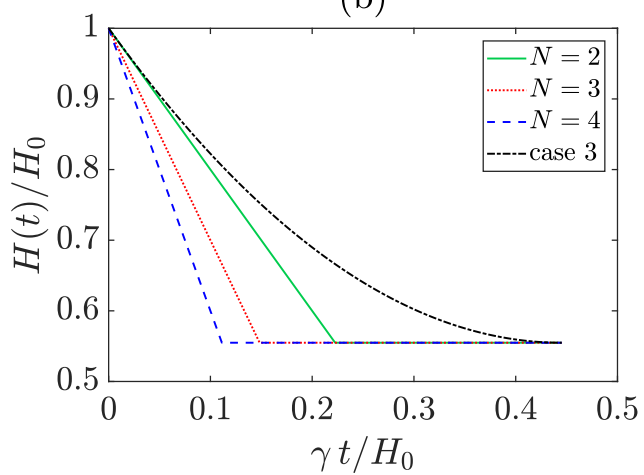

(d)

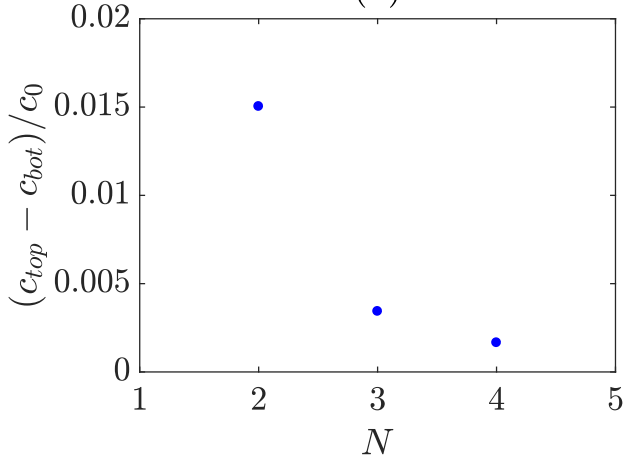

Figure 7: Panel (a) shows the optimal time-dependent drying rates for different values of $N$, and (b) depicts the evolution of the position of the evaporation surface for these optimal drying rates. Panel (c) shows the optimised concentration profiles at the end of the drying process. Panel (d) shows the value of the objective function, $\mathcal{J}$, for different values of $N$.

\section{Acknowledgments}

We are thankful to G. Goward, I. Halalay, X. Huang, M. Jiang, K. J. Harris, M. Z. Tessaro, H. Liu and S. Schougaard for helpful discussions. Funding for this research was provided by the Natural Sciences and Engineering Research Council of Canada (Collaborative Research \& Development grant CRDPJ 494074) and by General Motors of Canada.

\section{References}

[1] L. Lu, X. Han, J. Li, J. Hua, M. Ouyang, A review on the key issues for lithium-ion battery management in electric vehicles, Journal of Power 
Sources 226 (2013) 272-288.

[2] J. B. Goodenough, Y. Kim, Challenges for rechargeable Li batteries, Chemistry of Materials 22 (3) (2010) 587-603.

[3] M. L. Lazar, B. Sloan, S. Carlson, B. L. Lucht, Analysis of integrated electrode stacks for lithium ion batteries, Journal of Power Sources 251 (2014) 476-479.

[4] M. Singh, J. Kaiser, H. Hahn, Thick electrodes for high energy lithium ion batteries, Journal of The Electrochemical Society 162 (7) (2015) A1196-A1201.

[5] B. Westphal, H. Bockholt, T. Günther, W. Haselrieder, A. Kwade, Influence of convective drying parameters on electrode performance and physical electrode properties, ECS Transactions 64 (22) (2015) 57-68.

[6] S. Jaiser, M. Müller, M. Baunach, W. Bauer, P. Scharfer, W. Schabel, Investigation of film solidification and binder migration during drying of Li-Ion battery anodes, Journal of Power Sources 318 (2016) 210-219.

[7] K. M. Kim, W. S. Jeon, S. H. C. I. J. Chung, Effect of mixing sequences on the electrode characteristics of lithium-ion rechargeable batteries, Journal of Power Sources 83 (1-2) (1999) 108-113.

[8] G.-W. Lee, J. H. Ryu, W. Han, K. H. Ahn, S. M. Oh, "Effect of slurry preparation process on electrochemical performances of $\mathrm{LiCoO} 2 \mathrm{com}-$ posite electrode", Journal of Power Sources 195 (18) (2010) 6049-6054.

[9] M. Baunach, S. Jaiser, S. Schmelzle, H. Nirschl, P. Scharfer, W. Schabel, Delamination behavior of lithium-ion battery anodes: Influence of drying temperature during electrode processing, Drying Technology 34 (4) (2016) 462-473.

[10] A. Y. Shenouda, H. K. Liu, Synthesis and electrochemical studies on $\mathrm{Li} 2 \mathrm{CuSnO} 4$ and $\mathrm{Li} 2 \mathrm{CuSnSiO} 6$ as negative electrode in the lithium batteries, ECS Transactions 25 (36) (2010) 75-89.

[11] M. C. Kornherr, Influence of the drying process with infrared radiation on the mechanical and electrical properties of electrodes for lithium-ion batteries, Bachelor's thesis, Technical University of Munich (2016). 
[12] S. Jaiser, J. Kumberg, J. Klaver, J. L. Urai, W. Schabel, J. Schmatz, P. Scharfer, "Microstructure formation of lithium-ion battery electrodes during drying - An ex-situ study using cryogenic broad ion beam slopecutting and scanning electron microscopy (Cryo-BIB-SEM)", Journal of Power Sources 345 (2017) 97-107.

[13] W. Bauer, D. Nötzel, Rheological properties and stability of nmp based cathode slurries for lithium ion batteries, Ceramics International 40 (3) (2014) 4591-4598.

[14] A. Kraytsberg, Y. Ein-Eli, Conveying Advanced Li-ion Battery Materials into Practice The Impact of Electrode Slurry Preparation Skills, Advanced Energy Materials 6 (21).

[15] M. Schmitt, M. Baunach, L. Wengeler, K. Peters, P. Junges, P. Scharfer, W. Schabel, Slot-die processing of lithium-ion battery electrodes coating window characterization, Chemical Engineering and Processing: Process Intensification 68 (2013) 32-37.

[16] M. Schmitt, P. Scharfer, W. Schabel, Slot die coating of lithium-ion battery electrodes: investigations on edge effect issues for stripe and pattern coatings, Journal of Coatings Technology and Research 11 (1) (2014) 57-63.

[17] D. L. Wood III, J. Li, C. Daniel, Prospects for reducing the processing cost of lithium ion batteries, Journal of Power Sources 275 (2015) 234242 .

[18] S. Jaiser, L. Funk, M. Baunach, P. Scharfer, W. Schabel, Experimental investigation into battery electrode surfaces: The distribution of liquid at the surface and the emptying of pores during drying, Journal of Colloid and Interface Science 494 (2017) 22-31.

[19] H. Hagiwara, W. J. Suszynski, L. F. Francis, A raman spectroscopic method to find binder distribution in electrodes during drying, Journal of Coatings Technology and Research 11 (1) (2014) 11-17.

[20] M. Muller, L. Pfaffmann, S. Jaiser, M. Baunach, V. Trouillet, F. Scheiba, P. Scharfer, W. Schabel, W. Bauer, Investigation of binder distribution in graphite anodes for lithium-ion batteries, Journal of Power Sources 340 (2017) $1-5$. 
[21] M. M. Forouzan, C.-W. Chao, D. Bustamante, B. A. Mazzeo, D. R. Wheeler, Experiment and simulation of the fabrication process of lithium-ion battery cathodes for determining microstructure and mechanical properties, Journal of Power Sources 312 (2016) 172-183.

[22] M. Stein, A. Mistry, P. P. Mukherjee, Mechanistic understanding of the role of evaporation in electrode processing, Journal of The Electrochemical Society 164 (7) (2017) A1616-A1627.

[23] S.-L. Chou, Y. Pan, J.-Z. Wang, H.-K. Liu, S.-X. Dou, Small things make a big difference: binder effects on the performance of $\mathrm{Li}$ and $\mathrm{Na}$ batteries, Physical Chemistry: Chemical Physics 16 (2014) 20347-20359.

[24] C. M. Cardinal, Y. D. Jung, K. H. Ahn, L. F. Francis, Drying regime maps for particulate coatings, AIChE Journal 56 (11) (2010) 2769-2780.

[25] R. W. Style, S. S. L. Peppin, Crust formation in drying colloidal suspensions, Proceedings of the Royal Society A 467 (2125) (2011) 174-193.

[26] A. K. Atmuri, S. R. Bhatia, A. F. Routh, Autostratification in drying colloidal dispersions: Effect of particle interactions, Langmuir 28 (5) (2012) 2652-2658.

[27] R. Trueman, E. L. Domingues, S. Emmett, M. Murray, A. Routh, Autostratification in drying colloidal dispersions: A diffusive model, Journal of Colloid and Interface Science 377 (1) (2012) 207 - 212.

[28] M. G. Hennessy, A. Munch, Dynamics of slowly evaporating solventpolymer mixture with deformable upper surface, IMA Journal of Applied Mathematics 79 (2014) 681-720.

[29] M. G. Hennessy, C. J. W. Breward, C. P. Please, A two-phase model for evaporating solvent-polymer mixtures, SIAM Journal on Applied Mathematics 76 (4) (2016) 1711-1736.

[30] Dortmund data bank, http://www.ddbst.com (2018).

[31] M. Mundhwa, S. Elmahmudi, Y. Maham, A. Henni, Molar heat capacity of aqueous sulfolane, 4-formylmorpholine, 1-methyl-2-pyrrolidinone, and triethylene glycol dimethyl ether solutions from (303.15 to 353.15$) \mathrm{k}$, Journal of Chemical \& Engineering Data 54 (10) (2009) 2895-2901. 
[32] C. Yang, W. Xu, P. Ma, Thermodynamic properties of binary mixtures of p-xylene with cyclohexane, heptane, octane, and n-methyl-2pyrrolidone at several temperatures, Journal of Chemical \& Engineering Data 49 (6) (2004) 1794-1801.

[33] M. H. Ryou, J. Kim, I. Lee, S. Kim, Y. K. Jeong, S. Hong, J. H. Ryu, T. S. Kim, J. K. Park, H. Lee, J. W. Choi, Mussel-inspired adhesive binders for high-performance silicon nanoparticle anodes in lithium-ion batteries, Adv. Mater. 25 (2013) 1571.

[34] D. A. G. Bruggeman, Berechnung verschiedener physikalischer konstanten von heterogenen substanzen, Annalen der Physik 416 (7) (1935) 636-664.

[35] R. Magalhaes, N. Duraes, M. Silva, J. Silva, V. Sencadas, G. Botelho, J. L. Gómez-Ribelles, S. Lanceros-Méndez, The Role of Solvent Evaporation in the Microstructure of Electroactive $\beta$-Poly(Vinylidene Fluoride) Membranes Obtained by Isothermal Crystallization, Soft Materials 9 (1) (2010) 1-14.

[36] D.-W. Chung, M. Ebner, D. R. Ely, V. Wood, R. E. Garcia, Validity of the Bruggeman relation for porous electrodes, Modelling and Simulation in Material Science and Engineering 21 (2013) 074009.

[37] L. Masaro, X. Zhu, Physical models of diffusion for polymer solutions, gels and solids, Progress in polymer science 24 (5) (1999) 731-775. 